\title{
VIABILIDADE DE INSERÇÃO DA GELEIA DE GUAPEVA (Pouteria cf. Guardneriana Radlk.) NA ALIMENTAÇÃO ESCOLAR DO MUNICÍPIO DE PALMAS - TOCANTINS
}

Feasibility of insertion of guapeva jam (Pouteria cf. Guardneriana Radlk.) in the school feed of Palmas - Tocantins municipality

Viabilidad de la inserción de gelatina de guapeva (Pouteria cf. Guardneriana Radlk.) en la alimentación escolar del municipio de Palmas - Tocantins

Rômulo Alves Morais ${ }^{1,2}$, Romilda Ramos da Silva ${ }^{1,3}$, Gabriela Fonsêca Leal*1,3, Camila Mariane da Silva Soares ${ }^{1,2}$, Maria Olíviados Santos Oliveira ${ }^{1,2}$, Glêndara Aparecida de Souza Martins ${ }^{1,2}$

${ }^{1}$ Laboratório de Cinética e Modelagem de Processos (LaCiMP) - Universidade Federal do Tocantins

${ }^{2}$ Programa de Pós-Graduação em Ciência e Tecnologia de Alimentos (PPGCTA -UFT) - Universidade Federal do Tocantins

${ }^{3}$ Discentes do curso de Engenharia de Alimentos da Universidade Federal do Tocantins.

*Correspondência: Laboratório de Cinética e Modelagem de Processos, Escola de Engenharia de Alimentos, Universidade Federal do Tocantins, Quadra 109 Norte, Avenida NS 15, ALCNO-14, Plano Diretor Norte, Palmas - TO, CEP: 77001-090.e-mail: gabrielaleal476@gmail.com

\section{RESUMO}

O Programa de Alimentação Escolar (PNAE) é responsável por suprir as necessidades nutricionais dos alunos durante a permanência no ambiente escolar, com o mínimo de 30\% de seus recursos financeiros destinados à compra de alimentos provenientes da agricultura familiar. A inserção de frutos do Cerrado na alimentação escolar contribui na diversificação do desenvolvimento local sustentável e na valorização de tradições alimentares, além de possuírem alto valor nutricional. Nesse contexto, objetivou-se a formulação e processamento de geleia a partir do fruto guapeva (Pouteria cf. Guardneriana Radlk), nativo do Cerrado brasileiro, e verificar a viabilidade da inserção da geleia de guapeva na alimentação escolar por teste de aceitação sensorial com crianças de uma escola pública de PalmasTocantins. As formulações de geleia foram otimizadas por análise sensorial com provadores não treinados e a formulação otimizada foi submetida aos testes de aceitação sensorial com 59 crianças de uma escola da rede pública municipal do $3^{\circ}$ ao $4^{\circ}$ ano, de Palmas, Tocantins. Os resultados para o teste foram "Adorei" (61,22\%), "Gostei" (26,53\%); "Indiferente" (8,16\%); "Detestei" (4,09\%) e "Não gostei” não obteve votos. Indicando boa aceitabilidade, abrindo possibilidades para a diversificação para os cardápios de merenda escolar.

Palavras-chave: Alimentação escolar; Análise sensorial; Nutrição infantil.

\section{ABSTRACT}

The School Feeding Program (PNAE) is responsible for meeting the nutritional needs of students while in school, with a minimum of $30 \%$ of their financial resources for the purchase of food from family farms. The insertion of Cerrado fruits in school feeding contributes to the diversification of sustainable local development and the 
valorization of food traditions, besides having a high nutritional value. In this context, the objective was to formulate and process jam from guapeva fruit (Pouteria cf. Guardneriana Radlk), native of the Brazilian Cerrado, and to verify the feasibility of inserting guapeva jam. in school feeding by sensory acceptance test with children of a public school in Palmas-Tocantins. The jam formulations were optimized by sensory analysis with untrained tasters and the optimized formulation was submitted to sensory acceptance tests with 59 children from a public school from 3 rd to 4th grade, from Palmas, Tocantins. The results for the test were "Loved" (61.22\%), "Liked" (26.53\%); "Indifferent" (8.16\%); "I hated it" (4.09\%) and "I didn't like it" didn't get votes. Indicating good acceptability, opening possibilities for diversification for school lunch menus.

Keywords: School feeding; Sensory analysis; Child nutrition.

\section{RESUMEN}

El Programa de Alimentación Escolar (PNAE) es responsable de satisfacer las necesidades nutricionales de los estudiantes mientras están en el entorno escolar, con un mínimo del $30 \%$ de sus recursos financieros para la compra de alimentos de la agricultura familiar. La inserción de las frutas Cerrado en la alimentación escolar contribuye a la diversificación del desarrollo local sostenible y la valorización de las tradiciones alimentarias, además de tener un alto valor nutricional. En este contexto, el objetivo era formular y procesar gelatina de fruta de guapeva (Pouteria cf. Guardneriana Radlk), nativa del Cerrado brasileño, y verificar la viabilidad de la inserción de la gelatina de guapeva. en alimentación escolar mediante prueba de aceptación sensorial con niños de una escuela pública en Palmas-Tocantins. Las formulaciones de gelatina se optimizaron mediante análisis sensoriales con catadores no entrenados y la formulación optimizada se sometió a pruebas de aceptación sensorial con 59 niños de una escuela pública de $3{ }^{\circ}$ a $4{ }^{\circ}$ grado, de Palmas, Tocantins. Los resultados de la prueba fueron "Me encantó" (61.22\%), "Me gustó" (26.53\%); "Indiferente" (8,16\%); "Lo odiè" (4.09\%) y "No me gustó" no obtuvieron votos. Indicando una buena aceptabilidad, abriendo posibilidades de diversificación para los menús de almuerzos escolares.

Descriptores: Alimentación escolar; Análisis sensorial; Nutrición Infantil.

\section{INTRODUÇÃO}

O consumo de frutas pela população está relacionado, principalmente pelos benefícios associados á saúde. Os frutos do Cerrado são altamente nutritivos e oferecem características únicas de cor, sabor e aroma (GARCIA et al., 2017), além de potenciais tecnológicos e agroindustriais, a produção de novos produtos com base em frutos regionais promove o desenvolvimento local, fomentando a economia; e ainda mais, supera a barreira da sazonalidade, disponibilizando o fruto durante todo o ano, facilitando sua inserção no mercado.

A alimentação adequada e saudável é fundamental no desenvolvimento do indivíduo, principalmente de crianças e adolescentes em idade escolar, além da formação de hábitos alimentares saudáveis, contribui no aprendizado e rendimento escolar (RAPHAELLI et al., 2018). Além disso, O estado nutricional de crianças e adolescentes representa a condição de vida de uma população e indica sua perspectiva de vida e saúde na vida adulta (ANJOS e SILVEIRA, 2017).

A inclusão de alimentos regionais e da sociobiodiversidade no cardápio das escolas contribui para o aumento da produção agrícola familiar e da valorização dos produtos locais (GIRARDI et al., 2018). No entanto o estímulo ao consumo de alimentos regionais no âmbito da alimentação escolar é uma tarefa complexa. Para isso, os métodos de análises são ferramentas importantes para a construção da composição química e aceitação sensorial desses alimentos, além de serem parâmetros essenciais para o desenvolvimento de novos produtos com base em matérias-primas pouco exploradas.

A alimentação escolar gratuita é garantida por meio do Programa Nacional de Alimentação Escolar 
(PNAE). Considerado um dos maiores programas de alimentação escolar do mundo, o PNAE se subsidia na Portaria Interministerial $n^{\circ} 1010$, de 08 de maio de 2006, a qual considera:

Art. $1^{\circ}$ - Institui as diretrizes para a Promoção da Alimentação Saudável nas Escolas de educação infantil, fundamental e nível médio das redes pública e privado, em âmbito nacional, favorecendo o desenvolvimento de áreas que promovam e garantam a adoção de práticas alimentares mais saudáveis no ambiente escolar.

As normativas da Portaria são fundamentadas nas ações de educação alimentar e nutricional, na variedade de alimentos fornecidos de forma a respeitar à cultura e hábitos alimentares locais, e contribuir para o crescimento e desenvolvimentos dos alunos (OLIVEIRA, et al., 2018).

Além disso, inserção da agricultura familiar na alimentação escolar, tornou-se obrigatório, a partir da Lei $\mathrm{n}^{\circ} 11.947 / 2009$, o uso de, no mínimo, $30 \%$ do fundo de recursos financeiros para aquisição de gêneros alimentícios.

Dessa forma, pelo PNAE, as comunidades agrícolas começaram a participar diretamente do fornecimento e distribuição de alimentos in natura, estimulando o desenvolvimento econômico e sustentável das comunidades, a redução da desigualdade social, da pobreza e da migração campocidade, pela redistribuição de renda aos agricultores familiares (FERIGOLLO et al., 2017).

Nesse contexto, objetivou-se a formulação e processamento de geleia a partir do fruto guapeva (Pouteria cf. Guardneriana Radlk), nativo do Cerrado brasileiro, e verificar a viabilidade da inserção da geleia de guapeva na alimentação escolar por teste de aceitação sensorial com crianças de uma escola pública de Palmas- Tocantins.

\section{MATERIAIS E MÉTODOS}

\section{Obtenção da matéria prima e processamento:}

As geleias de guapeva foram preparadas utilizando frutos colhidos conforme o estágio de maturação, na cidade de Palmas - Tocantins e encaminhados para o Laboratório de Cinética e Modelagem de Processos, na Universidade Federal do Tocantins - Campus Palmas, onde foram processados conforme os seguintes procedimentos: descascamento/despolpamento/extração do suco, adição de água (se necessário), dissolução prévia da pectina, formulação (adição de açúcar, pectina e ácido), concentração à pressão atmosférica até $65^{\circ}$ Brix, enchimento a quente/fechamento da embalagem e rotulagem/armazenamento. A fonte de pectina foi extraída do albedo de maracujá de acordo com a metodologia descrita por Silva et al. (2012).

\section{Metodologia de Superfície de Resposta (MSR):}

As formulações foram preparadas com diferentes proporções de polpa e açúcar, albedo e ácido cítrico conforme a metodologia de superfície de resposta com planejamento fatorial completo $2^{3}$ (BOX e DRAPER, 1987).

\section{Análises microbiológicas:}

As formulações foram submetidas às análises microbiológicas segundo preconiza a RDC n ${ }^{\circ} 12$ de 02 de janeiro de 2001 que dispõe sobre Regulamento Técnico sobre os Padrões Microbiológicos para Alimentos e estabelece que geleias devem ser analisadas quanto a bolores e leveduras. O objetivo é detectar principalmente as leveduras osmofílicas que podem se desenvolver em alimentos com alta concentração de açúcar, entretanto também foram avaliados os patógenos alimentares Staphylococcus aureus, Salmonella typhimurium, coliformes totais a 
fim de garantir um alimento seguro ao consumo humano (BRASIL, 2001).

\section{Análise sensorial:}

Realizou-se um teste aceitação sensorial com provadores não-treinados, em cabines individuais, com amostras codificados com números de três dígitos, segundo a metodologia de Stone e Sidel (2005), para determinar a formulação ótima.

Os resultados do teste de aceitação serão avaliados por múltipla comparação de médias, utilizando o teste de Tukey a $5 \%(\mathrm{p}<0,05)$ utilizando o programa SISVAR (sistema de análise de variância para dados balanceados) (FERREIRA, 1999).

\section{Inserção da geleia na alimentação escolar:}

Para a viabilidade da aplicação da geleia de guapeva na alimentação escolar foi aplicado um teste de aceitação sensorial utilizando ficha com escala hedônica de expressão facial com cinco categorias para crianças de uma escola pública municipal de Palmas- TO, utilizando a formulação otimizada.

As geleias serão submetidas ao teste de aceitação em uma sala própria da escola, realizando a avaliação em um aluno por vez. Cada prova será feita em cabines individuais, tipo urna, sendo que o provador será auxiliado pelos pesquisadores para o preenchimento das respostas. O julgamento sensorial avaliara se o aluno desgostou, não gostou, indiferente, gostei e adorei, figura 01 .

Figura 1. Ficha de analise sensorial de escala facial para crianças do terceiro e quarto ano. Marque a carinha que mais representa o que você achou do

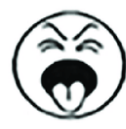

Detestei

1

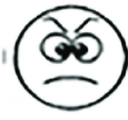

Não gostei

2

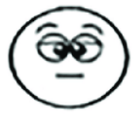

Indiferente

3

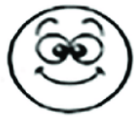

Gostei

4

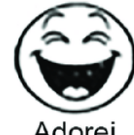

5
Os provadores avaliaram a aceitação das amostras através de uma escala hedônica facial estruturada mista de 5 pontos variando de 1 ("Super ruim") a 5 ("Super bom"), adaptada de Resurreccion (2004).

Cada julgador recebeu uma porção de cada amostra (aproximadamente $10 \mathrm{~g}$ ), acompanhado de biscoito cream cracker, em copos plásticos descartáveis brancos, codificados com números de três dígitos, de forma casualizados e balanceada, acompanhados de um copo de água para realização do branco entre as amostras. As formulações foram oferecidas aos julgadores de forma aleatória.

\section{Questões éticas:}

Para que a análise sensorial fosse realizada, o projeto foi submetido à apreciação do Comitê de Ética da Universidade Federal do Tocantins ao qual foi aprovado com Certificado de Apreciação Ética número 93357718.3.0000.5519 e parecer número 3.096.189. Entretanto, como critérios de exclusão, foram considerados os seguintes fatores: possuir alergia a algum ingrediente utilizado na elaboração da geleia, não ser aluno da escola em questão ou não entregar o Termo de Consentimento Livre e Esclarecido (TCLE) assinado pelo responsável legal.

\section{RESULTADOS E DISCUSSÃO}

As formulações de geleias foram processadas a partir da polpa de guapeva conforme a metodologia e apresentaram-se dentro dos padrões estabelecidos segundo as análises microbiológicas pela $\operatorname{RDC~}^{\circ} 12$, de 02 de Janeiro de 2001, determina o valor de limite para bolores e leveduras e Salmonella spp, além de ausência de contaminação por coliformes totais e Staphylococcus aureus, indicando um processamento asséptico, garantindo a inocuidade do produto para o consumo humano. 
A otimização da geleia foi realizada por análise sensorial com provadores não treinados resultando na melhor formulação com as seguintes características: menor razão polpa/açúcar e adição de albedo de maracujá como fonte de pectina, pois além de apresentar as melhores características sensoriais, foi a formulação de maior rendimento.

A formulação otimizada foi submetida aos testes de aceitação sensorial com 59 crianças de uma escola da rede pública municipal do $3^{\circ}$ ao $4^{\circ}$ ano, de Palmas, Tocantins.

Os resultados para o teste estão apresentados na figura (Fig. 2), abaixo:

Figura 2. Resultados para o teste de aceitação sensorial de crianças de uma escola da rede pública municipal de $3^{\circ}$ e $4^{\circ}$ ano, realizados para a formulação otimizada de geleia de guapeva, na cidade de Palmas, Tocantins, Brasil.

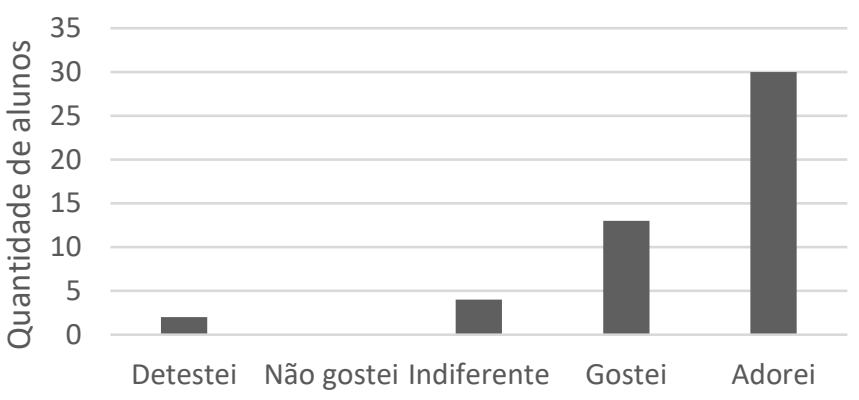

Entre o total de participantes que responderam o teste de escala hedônica fácil, a categoria "Adorei" foi a mais escolhida com $61,22 \%$, seguida da categoria "Gostei" com 26,53\%,; "Indiferente" com 8,16\%; "Detestei" com 4,09\% e "Não gostei" não obteve votos.

A aceitação de um alimento pelo aluno é principal fator para determinar a qualidade da alimentação servida pelas escolas.

Segundo a Resolução /CD/FNDE n ${ }^{\circ}$ 32, de 10 de agosto de 2006 que estabelece as normas para a execução do Programa Nacional de Alimentação Escolar, quanto ao controle de qualidade do programa, para metodologia de aceitabilidade, a qual deve ser definida pela Entidade Executora, observando os parâmetros técnicos, científicos e sensoriais reconhecidos; e o índice de aceitabilidade não deve ser a inferior a $85 \%$., logo a soma das categorias "Adorei" e "Gostei" resultam em 87,75\%, logo pode indicar a aceitação pelos provadores.

\section{CONCLUSÃO}

Os frutos do Cerrado exercem um papel essencial na busca do desenvolvimento sustentável e segurança alimentar da região. Frutos como a guapeva podem ser servidos de diversas formas, além de acompanharem outros cardápios, permitem a diversificação de pratos que atendam as necessidades nutricionais dos estudantes como contribuem para a valorização da região.

\section{AGRADECIMENTO}

Ao Laboratório de Cinética e Modelagem de Processos- LaCiMP- da Universidade Federal do Tocantins, Campus Palmas.

Todos os autores declararam não haver qualquer potencial conflito de interesses referente a este artigo.

\section{REFERÊNCIAS}

ANJOS, L. A dos; SILVEIRA, W. D. B. da. Estado nutricional dos alunos da Rede Nacional de Ensino de Educação Infantil e Fundamental do Serviço Social do Comércio (Sesc), Brasil, 2012. Ciência \& Saúde Coletiva, v. 22, p. 1725-1734, 2017.

BRASIL. AGÊNCIA NACIONAL DE VIGILÂNCIA SANITÁRIA (ANVISA). RDC 12 de 2 de Janeiro de 2001. Dispõe sobre Regulamento Técnico sobre os Padrões Microbiológicos para Alimentos. Diário Oficial da União. Brasília, 2001.

BRASIL. FUNDO NACIONAL DE DESENVOLVIMENTO DA EDUCAÇÃO (FNDE). Resolução/CD/FNDE No 32, de 10 de 
agosto de 2006. Estabelece as normas para a execução do Programa Nacional de Alimentação Escolar. Disponível em:< https://www.fnde.gov.br/>. Acesso em: $18 \mathrm{de}$ outubro de 2019.

BRASIL. MINISTÉRIO DA SAÚDE (MS); MINISTÉRIO DA EDUCAÇÃO (MEC). Portaria Interministerial n. ${ }^{\circ} 1.010$, de 08 de maio de 2006. Diário Oficial da União, 2006.

BRASIL. Lei no 11.947, de 16 de junho de 2009. Dispõe sobre o atendimento da alimentação escolar e do Programa Dinheiro Direto na Escola aos alunos da educação básica. Diário Oficial da União, 2009.

BOX, G. E. P.; DRAPER, N. R. Empirical model-building and response surfaces. Wiley, New York, 669 p., 1987.

DUTCOSKY, S. D. Análise sensorial de alimentos. In: Análise sensorial de alimentos. 2011.

FERIGOLLO, D.; KIRSTEN, V.R.; HECKLER, D.; FIGUEREDO, O.A.F.; PEREZCASSARINO, J.; TRICHES, R.M. Aquisição de produtos da agricultura familiar para alimentação escolar em municípios do Rio Grande do Sul. Revista de Saúde Pública, v. 51, p. 1-10, 2017.

GARCIA, L.; DAMIANI, C.; BECKER, F. Geleia de buriti (Mauritia flexuosa): agregação de valor aos frutos do Cerrado brasileiro. Braz. J. Food. Techno., Campinas, v. 20, e2016043, 2017.

GIRARDI, M. W., FABRI, R. K., BIANCHINI, V. U., MARTINELLI, S. S., \& CAVALLI, S. B.
Oferta de preparações culinárias e alimentos regionais e da sociobiodiversidade na alimentação escolar: um estudo na Região Sul do Brasil. Segurança Alimentar e Nutricional, v. 25, n. 3, p. 29-44, 2018.

FERREIRA, D. F. Sistema para análise de variância para dados balanceados - SISVAR. Lavras: UFLA, 1999. 92 p.

OLIVEIRA, E. H. S.; MORAIS, R. A. OLIVEIRA, T. T. B.; MARTINS, G. A. S. Cajuí (Anacardium Othonianum Rizz). In: Frutos do Cerrado: características e aplicações tecnológicas. / Adriana Régia Marques de Souza, Clarissa Daminani, Glêndara Aparecida de Souza Martins, Juliana Fonseca Moreira da Silva (organizadoras) - Curitiba: CRV, 2018. 148p.

SILVA, I. G., MARTINS, G. A. D. S., BORGES, S. V., MARQUES, G. R., \& REGIS, I. S. Influence of passion fruit albedo, citric acid, and the pulp/sugar ratio on the quality of banana preserves. Ciência Tecnologia Alimentos, Campinas, v.32, n.12, p.267-273, 2012.

RAPHAELLI, C. D. O.; PASSOS, L. D. F.; COUTO, S. D. F.; HELBIG, E. \& MADRUGA, S. W. Adhesion and acceptability of elementary school menus in rural schools. Brazilian Journal of Food Technology, Campinas, v. 20, e2016112, 2017.

RESURRECCION, A. V. A. Sensory aspects of consumer choices for meat and meat products. Meat Science, v. 66, n. 1, p. 11-20, 2004. STONE, H.; SIDEL, J. Sensory evaluation practices. 3rd. ed. New York: Academic Press, 2005. 377 p. 